\title{
Fucosylated CD147 promotes the malignant progression of ovarian cancer and is associated with glycolysis
}

Huimin Wang ( $\nabla$ wanghuimin2016@126.com )

Liaoning Cancer Institute and Hospital https://orcid.org/0000-0003-2363-4957

Yanyan Wang

Shengjing Hospital of China Medical University

Mingjun Zheng

Shengjing Hospital of China Medical University

Juanjuan Liu

Shengjing Hospital of China Medical University

Bei Lin

Shengjing Hospital of China Medical University

Research

Keywords: Lewis y, CD147, ovarian cancer, gene expression profile, glycolysis

Posted Date: April 14th, 2021

DOI: https://doi.org/10.21203/rs.3.rs-415869/v1

License: (a) (i) This work is licensed under a Creative Commons Attribution 4.0 International License. Read Full License 


\section{Abstract \\ Background}

This study investigated the molecular structural relationship between CD147 and Lewis y antigen in ovarian cancer cells, and explored the molecular mechanisms by which Lewis y leads to the malignant progression of ovarian cancer.

\section{Methods}

The expression of CD147 and Lewis y in three epithelial ovarian cell lines (RMG-I, COC1 and H08910) and their sub-lines (RMG-I-H, COCl/DDP and H08910/PM) with high metastatic potential and chemotherapy resistance was detected by quantitative real-time PCR, immunocytochemistry, and western blotting. The structural relationship between Lewis y and CD147 was determined by immunoprecipitation. Gene expression enrichment analysis was performed to elucidate the possible role of CD147 in the response to Lewis y in ovarian cancer.

\section{Results}

The expression of CD147, Lewis y, and FUT1 mRNA was significantly lower in ovarian cancer cell lines than in cells with a higher malignancy grade. Lewis y was an important component of CD147, and was predominantly expressed in the highly glycosylated form of CD147. Genes associatd with the CD147mediated response to Lewis y were mainly involved in cytokine-mediated signaling pathways and hexose metabolic processes. The expression of IL1A (IL-1a), which was highest in ovarian cancer, was significantly higher than in borderline, benign and normal ovarian tissues, and it was positively correlated with Lewis y in ovarian cancer.

\section{Conclusion}

CD147 was modified by fucosylation, and the effect of fucosylated CD147 on promoting the malignant progression of ovarian cancer may be related to glycolysis.

\section{Background}

Ovarian cancer is a tumor of the female genital tract that is associated with high mortality. The rate of early diagnosis is low, and more than $80 \%$ of cases are diagnosed at an advanced stage, which increases the likelihood of metastasis and poor prognosis[1]. Glycosyl antigens, which are widely expressed on the cell membrane, are important components of glycoproteins and glycolipids, and changes in these antigens are closely related to malignant progression, invasion, and metastasis of cells as well as other biological characteristics[2]. Ovarian cancer is characterized by changes in type II saccharide chains, and 
overexpression of the Lewis y antigen to different degrees is detected in more than $75 \%$ of epithelial ovarian cancer cases. Lewis y antigen is a di-fucosylated oligosaccharide and it is the terminal structure for saccharide chain synthesis. The a1,2-fucosyltransferase (FUT1) is a key enzyme in the synthesis of Lewis y antigen. The expression of Lewis y increases during the cancerization of cells, and plays an important role in the incidence, development, invasion, and metastasis of ovarian cancer [3].CD147, also known as EMMPRIN or Basigin, is expressed at high levels in many types of tumor cells and is involved in tumor metastasis and drug tolerance, and is related to the malignant progression of tumors[4], glycosylation plays a key role in the function of CD147 [5]. In previous studies, we showed that the positive expression rate and expression level of CD147 and Lewis y antigen are significantly higher in chemotherapy resistant than in sensitive ovarian cancer, and the expression of the two molecules is positively correlated[6].

In this study, we detect the expression of CD147 and Lewis y antigen to define the relationship between them in ovarian cancer. Functional enrichment analysis was performed on the basis of gene expression profiles to identify genes associated with CD147 in response to Lewis y antigen in ovarian cancer.

\section{Materials And Methods}

Cell lines and cell culture

The cell line RMG-I was derived from ovarian clear-cell carcinoma tissues, and RMG-I-H is a cell line with high expression levels of FUT1 and Lewis y antigen. The cells were maintained in DMEM supplemented with $10 \%$ fetal bovine serum (Hyclone, Logan, UT, USA). The highly metastatic human ovarian cell line H08910/PM and its parental line H08910, and the drug-tolerant human ovarian cell line COC1/DDP and its parental line COC1 were purchased from Shanghai Cell Resource Center of Chinese Academy of Sciences and cultured in RPMI-1640 medium containing 10\% fetal bovine serum. Cells were incubated at $37^{\circ} \mathrm{C}$ in an incubator with $5 \% \mathrm{CO}_{2}$.

Immunocytochemistry

Monolayer cell slides prepared from six ovarian cancer cell lines were fixed with $4 \%$ paraformaldehyde for 30 min and then stained according to the instructions for the Streptavidin-biotin complex (SABC) kit (Boshide Biotech Co., Wuhan, China). The reagents used were the same as those used in the immunohistochemistry assay. The antibodies used were mouse anti-human EMMPRIN (8D6) (1:100, Santa Cruz Biotechnology, Inc., Dallas, TX, USA) and mouse anti-human Lewis y antigen (1:100, Abcam, Cambridge, UK). The presence of brownish-yellow granules in the cytoplasm and cell membrane were considered a positive result.

Quantitative real-time PCR

Total RNA from treated cells was isolated using the TRIzol reagent (Life Technologies, Inc., Rockville, $\mathrm{MD}$ ). Complementary DNA (CDNA) was synthesized using the real-time polymerase chain reaction (RT- 
PCR) kit (Invitrogen, China) according to the manufacturer's protocol. The cDNA was subjected to realtime PCR analysis using the SYBR Green PCR Master Mix (Takara Bio., Dalian, China) on the ABI Prism 7500 Sequence Detection System (Applied Biosystems, Foster City, CA, USA). Primers for target genes were commercially synthesized (Table 1). GAPDH was used as the internal reference, and the LightCycler PCR detection system (Roche Diagnostics, Mannheim, Germany) was used for real-time PCR amplification. The Ct values for different templates were examined. After the amplification, solubility curve analysis was carried out and the fold changes of target gene expression levels were calculated using the $2^{-\triangle \Delta C T}$ method.

Table 1

Primer sequences used for quantitative real-time PCR

\begin{tabular}{|c|c|}
\hline Gene name & Primer sequence $\left(5^{\prime}-3^{\prime}\right)$ \\
\hline \multirow[t]{2}{*}{ FUT1 } & F: AGGTATAAACACACCCTCTGTGCTT \\
\hline & R: GAGTTCAGGGACAGACAGTGGTT \\
\hline \multirow[t]{2}{*}{ CD147 } & F: GACTGGGTACAAGATCAC \\
\hline & R: GCCTCCATGTTCAGGTTCTCAA \\
\hline \multirow[t]{2}{*}{ ALDOA } & F: TCATCCTCTTCCATGAGACACTCT \\
\hline & R: ATTCTGCTGGCAG ATACTGGCATA A \\
\hline \multirow[t]{2}{*}{ ENO1 } & F: TGCGTCCACTGGCATCTAC \\
\hline & R: CAGAGCAGGCGCAATAGTTTTA \\
\hline \multirow[t]{2}{*}{ STAT3 } & F: CATATGCGGCCAGCAAAGAA \\
\hline & R: ATACCTGCTCTGAAGAAACT \\
\hline \multirow[t]{2}{*}{ IL1A } & F: AGATGCCTGAGATACCCAAAACC \\
\hline & R: CCAAGCACACCCAGTAGTCT \\
\hline \multirow[t]{2}{*}{ GAPDH } & F: CCTTCATTGACCTCСАСТАC \\
\hline & R: GTTGTCATACTTCTCATGGTTC \\
\hline
\end{tabular}

Immunoprecipitation and western blotting

The samples were rinsed with cold PBS followed by incubation in 1\% Triton X-100 lysis buffer (Beyotime Biotechnology, Jiangsu, China). The cells were then centrifuged at $4^{\circ} \mathrm{C}$ at $14,000 \times \mathrm{g}$ for $15 \mathrm{~min}$. The supernatant was collected, and proteins were quantified using the BCA protein assay kit (Beyotime biotechnology, Jiangsu, China). Equal amounts of protein were separated by SDS-PAGE, electrotransferred onto PVDF membranes (Millipore, Bedford, MA, USA), and blocked in $5 \%$ bovine serum 
albumin/TBS (20 mM Tris-HCl, pH 7.4, $150 \mathrm{mM} \mathrm{NaCl}$, and 0.1\% Tween 20). The membranes were incubated with primary antibody $(1: 100)$ followed by secondary antibody $(1: 2,000)$, and bands were detected by chemiluminescence (ECL; ECL Prime Western Blotting Detection Reagent, Amersham, Pittsburgh, PA, USA) using the Molecular Imager system GDS8000b (UVP, Inc., Upland, CA, USA). Equal amounts of total protein $(1,000 \mu \mathrm{g})$ were mixed with EMMPRIN antibody, incubated at $4^{\circ} \mathrm{C}$ overnight, and then $40 \mu \mathrm{L}$ Protein A/G PLUS-Agarose was added and the mixture was shaken slowly at $4^{\circ} \mathrm{C}$ for $3-4 \mathrm{~h}$. The mixture was then centrifuged at 2,500 $\times \mathrm{g}$ to precipitate CD147. The immune precipitates were rinsed with lysis buffer three times, and the products were separated by $10 \%$ SDS-PAGE followed by western blotting using the procedures described above. Lewis y monoclonal antibody (1:500) was used to detect the expression of Lewis y on CD147.

Functional analysis and signaling pathway enrichment analysis

The differentially expressed genes associated with CD147, including CD147, were selected from our previous chip and analyzed using the Metascape database (http://metascape.org). Pathway and biological process enrichment analysis was performed with the following ontology sources: Kyoto Encyclopedia of Genes and Genomes (KEGG) Pathway, Gene ontology (GO), Biological Processes, Reactome Gene Sets, Canonical Pathways and CORUM. Terms with a $P$-value $<0.01$, minimum count of 3 , and enrichment factor $>1.5$ (enrichment factor is the ratio between observed count and the count expected by chance) were obtained and grouped into clusters according to their similarities. $P$-values were calculated based on accumulative hypergeometric distribution. The most statistically significant term within a cluster was selected as representative of the cluster.

Construction of protein interaction network and module screening

Protein-protein interaction (PPI) network analysis was performed using the Search Tool for the Retrieval of Interacting Genes (STRING, http://string. embl.de/) database and visualized using Cytoscape. A confidence score $\geq 0.4$ was set as the cut-off criterion. Then, the Molecular Complex Detection (MCODE) was performed to screen modules of PPI networks with a degree cutoff $=2$, node score cutoff $=0.2, \mathrm{k}$ core $=2$, and max. depth $=100$.

Immunohistochemical analysis of paraffin-embedded tissues

To evaluate differentially expressed genes at the protein level, the expression of IL-1a and Lewis y in ovarian tissue samples was detected by immunohistochemical staining. A total of 137 paraffinembedded ovarian tissue samples were obtained from operations performed between 2002 and 2013 in the Department of Gynecology of Shengjing Hospital of China Medical University, including 99 cases of primary ovarian epithelial carcinoma, 12 cases of ovarian borderline tumor, 16 cases of ovarian benign tumor, and 20 normal ovarian tissues. No patient received chemotherapy or radiation therapy before surgery. All the pathologies were incipient and the clinicopathological data were complete. Streptavidinperoxidase kits were used according to the manual. The positive controls were colon cancer sections with known Lewis y positive expression and gastric cancer sections with positive IL-1a expression, whereas 
the negative controls were generated by omission of the primary antibody or incubation with an isotype control antibody. The primary antibodies against Lewis y (Abcam, Cambridge, UK) and IL-1a (Santa Cruz Biotechnology, Inc., Dallas, TX, USA) were used at a concentration of 1:100. A sample was considered positive when buffy granules were detected in the cell membrane and cytoplasm. Two observers read the sections to control error, independently.

Statistical analysis

SPSS 22.0 software (IBM, Armonk, NY, USA) was used for statistical analyses. Quantitative data were presented as the mean $\pm S D$. Quantitative real-time PCR results were expressed as the mean $\pm S E M$. Positive ratios were evaluated using the chi-square $(\chi 2)$ test. The Student's $t$ test was used for comparisons between two groups, and one-way ANOVA with LSD or Bonferroni post hoc test was used for comparisons between more than two groups. The correlation coefficient $r$ between IL-1a and Lewis y was calculated using Spearman's correlation analysis. A $P$ value of $<0.05$ was considered statistically significant.

\section{Results}

Immunocytochemical detection of the expression of CD147 and Lewis y in three groups of ovarian cancer cells with varying degrees of malignancy

Immunocytochemical analysis showed positive CD147 staining in RMG-I-H, H08910/PM, and COC1/DDP cells, as indicated by the detection of buffy particles in the cytoplasm and cell membrane; the cumulative optical density values were $66.91 \pm 6.45,38.83 \pm 1.43$, and $31.92 \pm 2.81$, respectively. CD147 positive stainig in RMG-I, H08910, and COC1 cells was detected as amber particles, with cumulative optical density values of $16.54 \pm 1.31,19.10 \pm 3.02$, and $4.49 \pm 0.13$, respectively. Clustering expression of CD147 in RMG-I-H, H08910/PM, and COC1/DDP cells was higher than that in the parental cell lines, and positive staining was significantly enhanced $(P<0.05$, Fig. $1 \mathrm{~A}$ and Table 2$)$. Lewis y localized to the cell membrane and cytoplasm, and positively stained particles were detected as buffy or brown in RMG-I-H, H08910/PM, and COC1/DDP cells; they were widely distributed, and the cumulative optical density values were $53.90 \pm 4.33,37.31 \pm 0.19$, and $28.52 \pm 1.45$, respectively. Lewis y positive staining in RMG-I, $\mathrm{HO8910}$, and $\mathrm{COC1}$ cells was detected as amber dispersed particles, and the cumulative optical density values were $22.18 \pm 0.64,14.96 \pm 0.61$, and $16.26 \pm 0.83$, respectively (Fig. 1B and Table 2). The expression of Lewis $y$ was similar to that of CD147 and was significantly lower than that in the corresponding RMG-IH, H08910/PM, and COC1/DDPP cells $(P<0.05)$. 
Table 2

The accumulative optical density values for CD147\Lewis y antigen in three groups of ovarian cancer cells with varying degrees of malignancy that were measured by using immuocytochemical methods

\begin{tabular}{|lll|}
\hline Cell line & CD147 & Lewis y \\
\hline RMG-I & $16.54 \pm 1.31$ & $22.18 \pm 0.64$ \\
RMG-I-H & $66.91 \pm 6.45^{\star}$ & $53.90 \pm 4.33^{\star}$ \\
HO8910 & $19.10 \pm 3.02$ & $14.96 \pm 0.61$ \\
COC1 & $38.83 \pm 1.43^{*}$ & $37.31 \pm 0.19 *$ \\
COC1/DDP & $4.49 \pm 0.13$ & $16.26 \pm 0.83$ \\
* $P<0.05$, RMG-I-H compared to RMG-I cells, H08910/PM compared to H08910 cells, and COC1/DDP \\
compared to COC1 cells.
\end{tabular}

Quantitative real-time PCR detection of the expression of CD147 and FUT1 in three groups of ovarian cancer cells with varying degrees of malignancy

Figure 2A shows that the expression level of CD147 mRNA was approximately $1.6-(P<0.05)$ and 3.5- $(P$ $<0.01)$ fold higher in COC1/DDP and H08910/PM cells than in COC1 and H08910 cells. The expression level of CD147 mRNA in RMG-I-H cells was approximately 0.62-fold lower than that in RMG-I cells $(P>$ 0.05). The results of real-time PCR confirmed that the expression level of FUT1 mRNA was 3.07-fold higher in RMG-I-H cells than in RMG-I cells, 2.41-fold higher in H08910/PM cells than in H08910 cells, and 2.72-fold higher in COC1/DDP cells than in COC1 cells ( $P<0.05$, Fig. 2B).

Western blot analysis of CD147 expression in three groups of ovarian cancer cells with varying degrees of malignancy

Figure 3 shows that the expression levels of the CD147 protein were significantly higher in the transfected line RMG-I-H, the highly metastatic line H08910/PM, and the drug tolerant line COC1/DDP than in the parental cell lines, and the expression levels of the highly glycosylated form of CD147 were $1.43,1.75$, and 1.81 fold higher than those in the original parent cells, respectively $(P<0.05)$. Compared with the highly glycosylated form, the low glycosylated form of CD147 did not change significantly. The expression levels of the low glycosylated form in H08910 and the highly metastatic line HO8910/PM were significantly higher than those in RMG-I, RMG-I-H, COC1, and COC1/DDP cells. This difference may be related to the different forms of MMPs induced by CD147 with different molecular weights [7]. We detected a protein band with a molecular weight of approximately $26 \mathrm{kDa}$ in the total protein lysates of the six cell lines analyzed. Because the CD147 core protein is $27 \mathrm{kDa}$, we assumed that it might be a membrane-detached form of CD147[8] or a subtype of CD147 basigin-3[9].

Determination of the correlation between Lewis y and CD147 in the six cell lines by immunoprecipitation 
Immunoprecipitation results (Fig. 4) showed that Lewis y antigen expression was detected in CD147 molecules from the six cell lines. Lewis y was mainly expressed in the highly glycosylated form and the unknown form of CD147. The expression levels of Lewis y in CD147 were 2.12, 1.82, and 2.33 fold higher in the transfected line RMG-I-H, the highly metastatic line H08910/PM, and the drug tolerant line COC1/DDP, respectively, than in the parental cell lines when the same amounts of CD147 antibody were used for the total proteins and the precipitates $(P<0.05)$. Lewis y antigen at high expression levels was also detected in CD147 with a molecular weight of about $26 \mathrm{kDa}$ in the six types of ovarian cancer cells.

Function and enrichment analysis of CD147 involved in the differentially expressed genes in response to Lewis y

Based on the Metascape database, the biological functions and pathways for the differentially expressed genes associated with CD147 from our previous chip described above were analyzed. A heatmap of enriched terms across input gene lists, colored by $P$-values, was generated. The results showed that genes were mainly enriched in cytokine-mediated signaling pathways, regulation of cell adhesion, regulation of T cell apoptotic process, and hexose metabolic process (Fig. 5). Subsequently, we selected a high degree of enrichment, grouped clustering according to the correlation of functional pathways, and constructed a network, with different colors representing different categories (Fig. 6).

Construction of a protein-protein interaction network and module screening

We also constructed a protein interaction network to determine how these genes interact with each other, as well as to find the central node of the PPI network. The PPI network is depicted in Fig. 7. The PPI network included 207 nodes and 369 edges. Each node represents a protein, and the edge between the nodes represents the interaction between the two proteins. The thickness of the line represents the strength of the relationship. A greater connectivity is represented by a larger node. As shown in the figure, the highly connected proteins in the network were encoded by the ALDOA, ENO1, STAT3, ACTG2, and ILIA genes. These genes are all related to glycolysis, suggesting that the malignant behavior of ovarian cancer enhanced by the glycosylation of CD147 may be related to glycolysis in cells.

Validation of the differentially expressed genes by quantitative real-time PCR

Four differentially expressed genes, ALDOA, ENO1, STAT3, and IL1A, were selected for quantitative realtime PCR analysis. The proteins encoded by these genes were highly connected in the PPI network. As shown in Fig. 8, the mRNA levels of the four genes were significantly higher in three ovarian cancer cell lines with high expression of Lewis y and CD147 (RMG-I-H, H08910/PM, and COC1/DDP cells) than in cells with a lower malignancy grade (RMG-I, HO8910, and COC1). This was consistent with the results of gene chip analysis.

Validation of protein expression by immunohistochemical staining

To confirm the gene expression results at the protein level, immunohistochemical staining for IL-1a and Lewis y antigen was performed on all paraffin embedded samples. Similar to Lewis y, IL-1a localized 
mainly to the membrane and cytoplasm (Fig. 9). The positive expression rates of IL-1 a in the malignant, borderline, benign, and normal groups were $80.81 \%, 66.67 \%, 43.75 \%$, and $30.00 \%$, respectively (Table 3 ). IL-1 a positive expression rate was highest in the malignant group and was significantly higher than the rate in the benign and normal groups $(P<0.01)$. The expression rate was higher in the malignant group than in the borderline group, although the difference was not statistically significant $(P>0.05)$. The positive expression rate of Lewis y was highest in the malignant group (86.87\%), which was significantly higher than that in the benign group (37.50\%) (Table 3).

Table 3

Expression of IL-1a and Lewis y antigen in various ovarian tissues

\begin{tabular}{|c|c|c|c|c|c|c|c|c|c|c|c|}
\hline \multirow[t]{2}{*}{ Groups } & \multirow[t]{2}{*}{$\mathrm{n}$} & \multicolumn{5}{|c|}{ IL-1a } & \multicolumn{5}{|c|}{ Lewis y } \\
\hline & & - & + & ++ & +++ & Positive (\%) & - & + & ++ & +++ & Positive (\%) \\
\hline Malignant & 99 & 19 & 21 & 32 & 27 & $80(80.81)^{a}$ & 13 & 19 & 34 & 33 & $86(86.87)^{a}$ \\
\hline Borderline & 12 & 4 & 1 & 6 & 1 & $8(66.67)^{c}$ & 4 & 2 & 5 & 1 & $8(66.67)^{b}$ \\
\hline Benign & 16 & 9 & 3 & 3 & 1 & $7(43.75)$ & 10 & 2 & 4 & 0 & $6(37.50)$ \\
\hline Normal & 10 & 7 & 3 & 0 & 0 & $3(30.00)$ & 10 & 0 & 0 & 0 & $0(0)$ \\
\hline \multicolumn{12}{|c|}{${ }^{a}$ Compared with the benign and normal group, both $P<0.01$. } \\
\hline \multicolumn{12}{|c|}{${ }^{\mathrm{b}}$ Compared with the benign group, $P<0.05$. } \\
\hline
\end{tabular}

Among the 99 cases of ovarian cancer, 53 and 26 cases showed simultaneous high or low expression patterns of both IL-1a and Lewis y antigen (Table 4). Spearman's correlation analysis revealed a positive correlation between the expression of IL-1a and Lewis y $(r=0.575, P=0.00003)$.

Table 4

The relevance of IL-1a and Lewis y expression in ovarian cancer samples

\begin{tabular}{|llll|}
\hline IL-1a & Lewis y & Total \\
\cline { 2 - 3 } & High $(++++++)$ & Low $(-,+)$ & \\
\hline High $(++,+++)$ & 53 & 6 & 59 \\
\hline Low $(-,+)$ & 14 & 26 & 40 \\
\hline Total & 67 & 32 & 99 \\
\hline Spearman correlation, $r=0.575, P=0.00003$ \\
\hline
\end{tabular}




\section{Discussion}

The structures of sacccharide chains in saccharide compounds on the cell surface are closely related to certain biological behaviors of tumor cells. In previous work, we showed that Lewis y antigen can promote metastasis and drug tolerance in tumor cells by regulating the expression of tumor-related

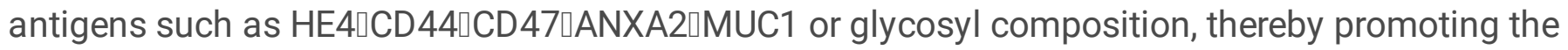
malignant transformation of ovarian cancer cells.

CD147 is an important protein that contains fucose structures on the cell surface, and it has important functions in the progression of tumors[4]. Our previous results showed that Lewis y antigen is an important component of CD147[10]. To confirm that the correlation between Lewis $y$ antigen and the CD147 structure is ubiquitous and is not dependent on cell type, we used two groups of human ovarian cancer cell lines, a highly metastatic line and its parental line (H08910/PM and H08910) and a drug tolerant line and its parental line (COC1/DDP and COC1). The results showed that the expression levels of Lewis $y$ antigen and CD147 in H08910/PM and COC1/DDP cells increased in correlation with the increase in the malignancy of tumor cells $(P<0.05)$, and the expression level of Lewis y antigen on the CD147 molecule also significantly increased $(P<0.05)$; these changes were regulated at the gene level. Lewis y antigen was mainly expressed on the CD147 molecule in a highly glycosylated form, which may be related to the fact that N-glycosylation of CD147 can inhibit ubiquitin-mediated degradation[11].

To clarify the molecular mechanisms underlying the role of Lewis y antigen in the development of ovarian cancer, we used the human genome oligonucleotide microarray technique to analyze the gene expression profiles of ovarian cancer cell lines with different degrees of Lewis y expression. We identified CD147 among the differentially expressed genes in response to Lewis y[6]. In this study, we demonstrated that the expression of CD147 and Lewis y antigen was positively correlated at the protein level; moreover, enrichment analysis including functional, signaling pathway analysis, and interaction network analysis provided crucial information for further investigation of CD147 in ovarian cancer. We found that differentially expressed genes were mainly associated with cytokine-mediated signaling pathway, regulation of cell adhesion, regulation of $\mathrm{T}$ cell apoptotic process, and hexose metabolic process. We constructed an interaction network map and predicted a large number of interacting genes, including ALDOA, ENO1, STAT3, ACTG2, and IL1A. Aldolase A (ALDOA), is an important enzyme that exists in almost all organisms and participates in glycolysis. ALDOA is involved in many cellular functions, such as the regulation of cell morphology and motion, and the synthesis of ATP. ALDOA is expressed at high levels in many malignant tumors, and its expression is related to differentiation, TNM stage, and prognosis[12]. Alpha-enolase (ENO1) belongs to the enolase family and is a key enzyme in the glycolysis process. The transcriptional activity and protein expression of ENO1 are increased in many tumors[13]. Increased ENO1 glycolytic activity and ATP citrate lyase expression in tumors suggests that ENO1 promotes tumor metabolism, and high ENO1 expression thus provides tumor cells with a growth advantage. Interleukin-1(IL-1a) is cytokine that is secreted by a variety of cells and functions in the regulation of immunity and metabolism. Studies show that increased expression of IL-1a in malignant tumors (such as gastric cancer, breast cancer, and colorectal cancer) promotes tumor proliferation and 
metastasis [14]. Continuous injection of IL-1a into mice decreases the activity of glycolytic enzymes, and the mice show metabolic changes associated with cancer cachexia[15]. These genes are all related to glycolysis, suggesting that the increase in the malignant behavior of ovarian cancer caused by the glycosylation of CD147 may be related to glycolysis. During the growth of tumor cells, glycolysis is activated as a result of oxygen deficiency and increased glucose uptake; the increase of blood glucose concentration induces the nonenzymatic glycosylation or glycosylation of proteins and leads to the accumulation of advanced glycosylation products[16]. Jones et al. showed that the ST6Gal-I sialyltransferase can modify N-glycan on the surface of tumor cells by sialylation, and promote the adaptation of tumor cells to a hypoxic environment[17]. These findings are consistent with our results. In previous work, we showed that the expression of TGF- $\beta 1$ and IL- 6 increases in correlation with the expression of Lewis y antigen in ovarian cancer cells (relevant experimental results are being collated and have not been published). Because Lewis y antigen is closely related to the interleukin family, we focused on IL-1a, and determined the expression of IL-1a and Lewis y in 127 cases of ovarian epithelial neoplasms and 10 cases of normal ovarian tissues by immunohistochemistry. The results showed that the positive expression rates of IL-1 $a$ and Lewis y increased in correlation with the change of normal ovarian tissues to malignant tissues, and the expression of the two molecules showed a significant positive correlation.Lewis y has been identified as an independent risk factor for the prognosis of ovarian cancer in clinical tissue samples in previous study[18]. The statistical results of this study showed that the survival rate of ovarian cancer was significantly correlated with the level of IL-1a expression in malignant tissues, as a higher expression of IL-1 a was associated with a worse prognosis. This suggested that IL-1 a is a prognostic indicator in ovarian malignant epithelial tumors. Analysis of a larger number of samples is necessary for further verification. These results indicated that the increase of Lewis $y$ antigen activated a series of signaling pathways leading to glycolysis and promoted tumor progression. Further comprehensive and in-depth studies are needed to provide additional evidence for the development of novel drugs and therapeutic strategies selectively targeting Lewis $y$ in ovarian cancer.

We used three groups of highly malignant cell lines and their parental cell lines to confirm that the modification of Lewis y antigen on the surface of highly glycosylated CD147 molecules increased the malignancy of cells, which may have important implications for the early diagnosis of ovarian tumors and the design of novel therapeutic strategies.

\section{Abbreviations}

a1,2-fucosyltransferase :FUT1

matrix metalloproteinase:MMP

Kyoto Encyclopedia of Genes and Genomes:KEGG

Gene ontology:GO

Protein-protein interaction:PPI

Page $11 / 21$ 
Molecular Complex Detection:MCODE

Aldolase A:ALDOA

Alpha-enolase:ENO1

Interleukin-1:IL-1a

\section{Declarations}

Ethics approval and consent to participate:Samples were fully encoded to protect patient confidentially. The study and its protocols were approved by the Research Ethics committees of Shengjing Hospital Affiliated with China Medical University (2013PS66K).

Consent for publication:Not applicable.

Availability of data and materials:Not applicable.

Competing interests:The authors declare that they have no conflict of interest.

Funding:This work was supported by grants from The National Natural Science Foundation of China (81172491, 81101527, 81472437, 81672590), and Outstanding Scientific Fund of Shengjing Hospital (no: 201303).

Authors' contributions:HW carried out most parts of the experiment; $\mathrm{YW}$ and $\mathrm{MZ}$ participated in the experiment; BL participated in the design of the study; HW and JL performed the statistical analysis. All authors read and approved the final manuscript.

Acknowledgements:Not applicable.

\section{References}

1. Mallen A, Soong TR, Townsend MK, Wenham RM, Crum CP, Tworoger SS. Surgical prevention strategies in ovarian cancer. Gynecol Oncol. 151(2018):166-175.doi: 10.1016/j.ygyno.2018.08.005.

2. Mihoko Sutoh Yoneyama, Yuki Tobisawa, Shingo Hatakeyama, Misaki Sato, Kiyoshi Tone, Yota Tatara, Ikuko Kakizaki, Tomihisa Funyu, Minoru Fukuda, Senji Hoshi, Chikara Ohyama, Shigeru Tsuboi.A mechanism for evasion of CTL immunity by altered O-glycosylation of HLA class I. J Biochem. 161(2017)479-492.doi:10.1093/jb/mvw096.

3. Zuofei Song, Qian Guo, Huimin Wang, Lingling Gao, Shuang Wang, Dawo Liu, Juanjuan Liu, Yue Qi, Bei Lin.miR-5193, regulated by FUT1, suppresses proliferation and migration of ovarian cancer cells by targeting TRIM11.Pathol Res Pract. 216(2020):153148.doi:10.1016/j.prp.2020.153148.

4. Hong Yang, Biliang Chen.CD147 in ovarian and other cancers.Int J Gynecol Cancer. 23(2013):28doi:10.1097/IGC.0b013e3182749139. 
5. Wei Tang, Sharon B Chang, Martin E Hemler.Links between CD147 function, glycosylation, and caveolin-1. Mol Biol Cell.15(2004)4043-4050. doi:10.1091/mbc.e04-05-0402.

6. Liancheng Zhu, Zhenhua Hu, Juanjuan Liu, Jian Gao, Bei Lin.Gene expression profile analysis identifies metastasis and chemoresistance-associated genes in epithelial ovarian carcinoma cells. Med Oncol.32(2015) 426. doi:10.1007/s12032-014-0426-5.

7. Joost P G Sluijter, Wilco P C Pulskens, Arjan H Schoneveld, Evelyn Velema, Chaylendra F Strijder, Frans Moll, Jean-Paul de Vries, Jan Verheijen, Roeland Hanemaaijer, Dominique P V de Kleijn, Gerard Pasterkamp.Matrix metalloproteinase 2 is associated with stable and matrix metalloproteinases 8 and 9 with vulnerable carotid atherosclerotic lesions: a study in human endarterectomy specimen pointing to a role for different extracellular matrix metalloproteinase inducer glycosylation forms. Stroke.37(2006)235-239. doi:10.1161/01.STR.0000196986.50059.e0

8. Nagayasu Egawa, Naohiko Koshikawa, Taizo Tomari, Kazuki Nabeshima, Toshiaki Isobe, Motoharu Seiki. Membrane type 1 matrix metalloproteinase (MT1-MMP/MMP-14) cleaves and releases a 22$\mathrm{kDa}$ extracellular matrix metalloproteinase inducer (EMMPRIN) fragment from tumor cells. J Biol Chem.281(2006)37576-37585. http://doi.org/10.1074/jbc.

9. Robert J Belton Jr, Li Chen, Fernando S Mesquita, Romana A Nowak.Basigin-2 is a cell surface receptor for soluble basigin ligand. J Biol Chem. 283(2008)17805-17814. doi:/10.1074/jbc.M801876200.

10. Zhenhua Hu, Mingbo Cai, Lu Deng, Liancheng Zhu, Jian Gao, Mingzi Tan, Juanjuan Liu, Bei Lin.The fucosylated CD147 enhances the autophagy in epithelial ovarian cancer cells.

Oncotarget.7(2016)82921-82932. doi:/10.18632/oncotarget.13289.

11. Wenjuan Wang, Qingquan Li, Jingda Xu, Xixi Cao, Haixia Li, Feng Tang, Qi Chen, Jinming Yang, Zude $\mathrm{Xu}$, Xiuping Liu.Interaction between CD147 and P-glycoprotein and their regulation by ubiquitination in breast cancer cells. Chemotherapy.54(2008) 291-301.doi:1159/000151225.

12. Chang YC, Yang YC, Tien CP, Yang CJ, Hsiao M.Roles of Aldolase Family Genes in Human Cancers and Diseases.Trends Endocrinol Metab. 29(2018)549-559.doi:1016/j.tem.2018.05.003.

13. Cappello P, Principe M, Bulfamante S,Novelli F.Alpha-Enolase (ENO1), a potential target in novel immunotherapies. Front Biosci (Landmark Ed).22(2017) 944-959.doi:2741/4526.

14. Bou-Dargham MJ, Khamis ZI, Cognetta AB, Sang QA.The role of interleukin-1 in inflammatory and malignant human skin diseases and the rationale for targeting interleukin-1 alpha. Med Res Rev.37(2017)180-216. doi:1002/med.21406.

15. Korekane H, Nishikawa A,Imamura K.Mechanisms mediating metabolic abnormalities in the livers of Ehrlich ascites tumor-bearing mice. Arch Biochem Biophys.412(2003)216-222.doi:1016/s00039861(03)00041-9.

16. Khan MI, Rath S, Adhami VM, Mukhtar H.Hypoxia driven glycation: Mechanisms and therapeutic opportunities. Semin Cancer Biol.49(2018)75-82. doi:1016/j.semcancer.2017.05.008.

17. Jones RB, Dorsett KA, Hjelmeland AB,Bellis SL.The ST6Gal-I sialyltransferase protects tumor cells against hypoxia by enhancing HIF-1a signaling. J Biol Chem. 293(2018)5659- 
5667.doi:1074/jbc.RA117.001194.

18. Liancheng Zhu, Huilin Feng, Shan Jin, Mingzi Tan, Song Gao, Huiyu Zhuang, Zhenhua Hu, Huimin Wang, Zuofei Song, Bei Lin.High expressions of BCL6 and Lewis y antigen are correlated with high tumor burden and poor prognosis in epithelial ovarian cancer.Tumour Biol.39(2017) 10104283. doi:1177/1010428317711655.

Figures 
A
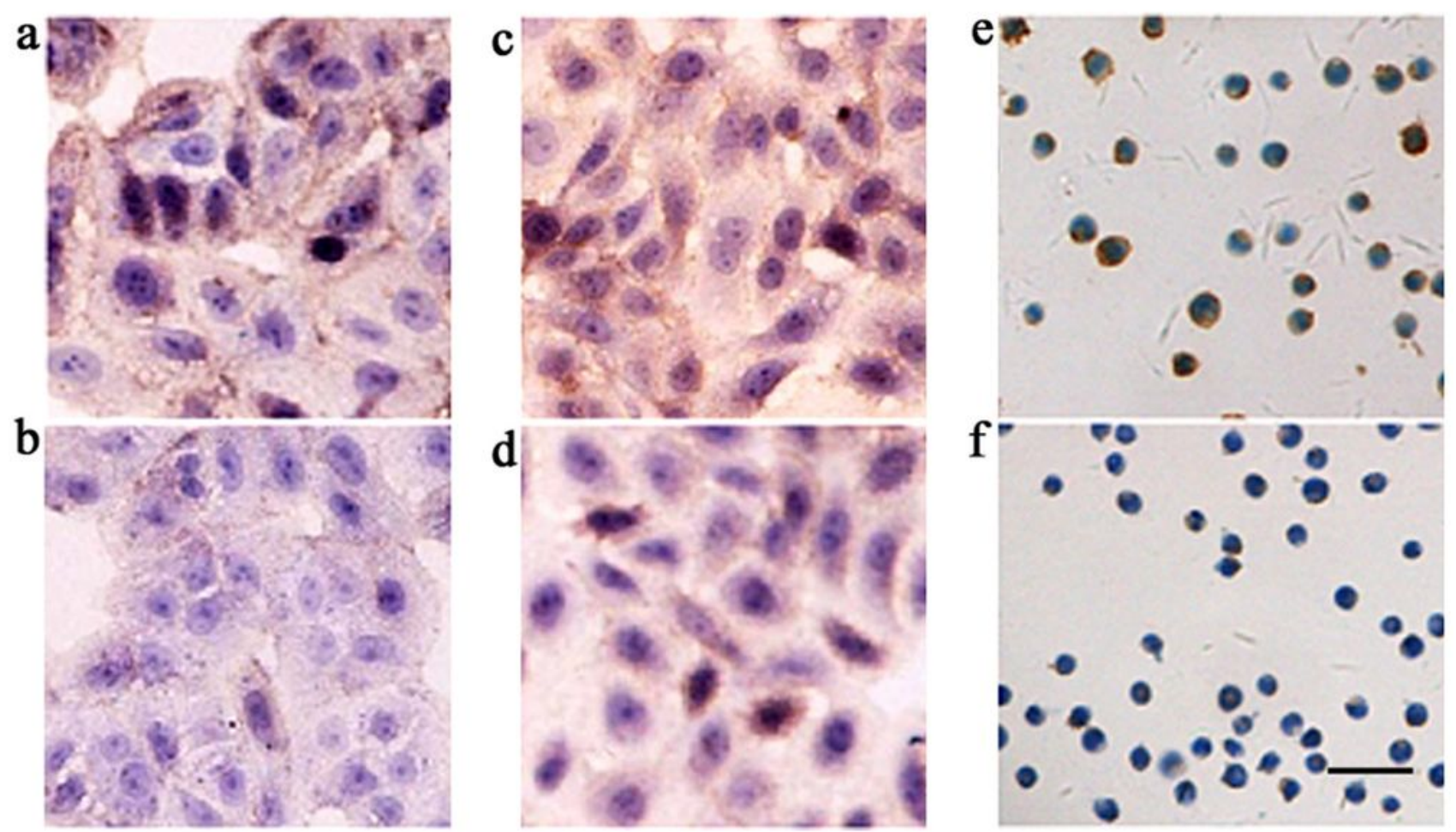

B
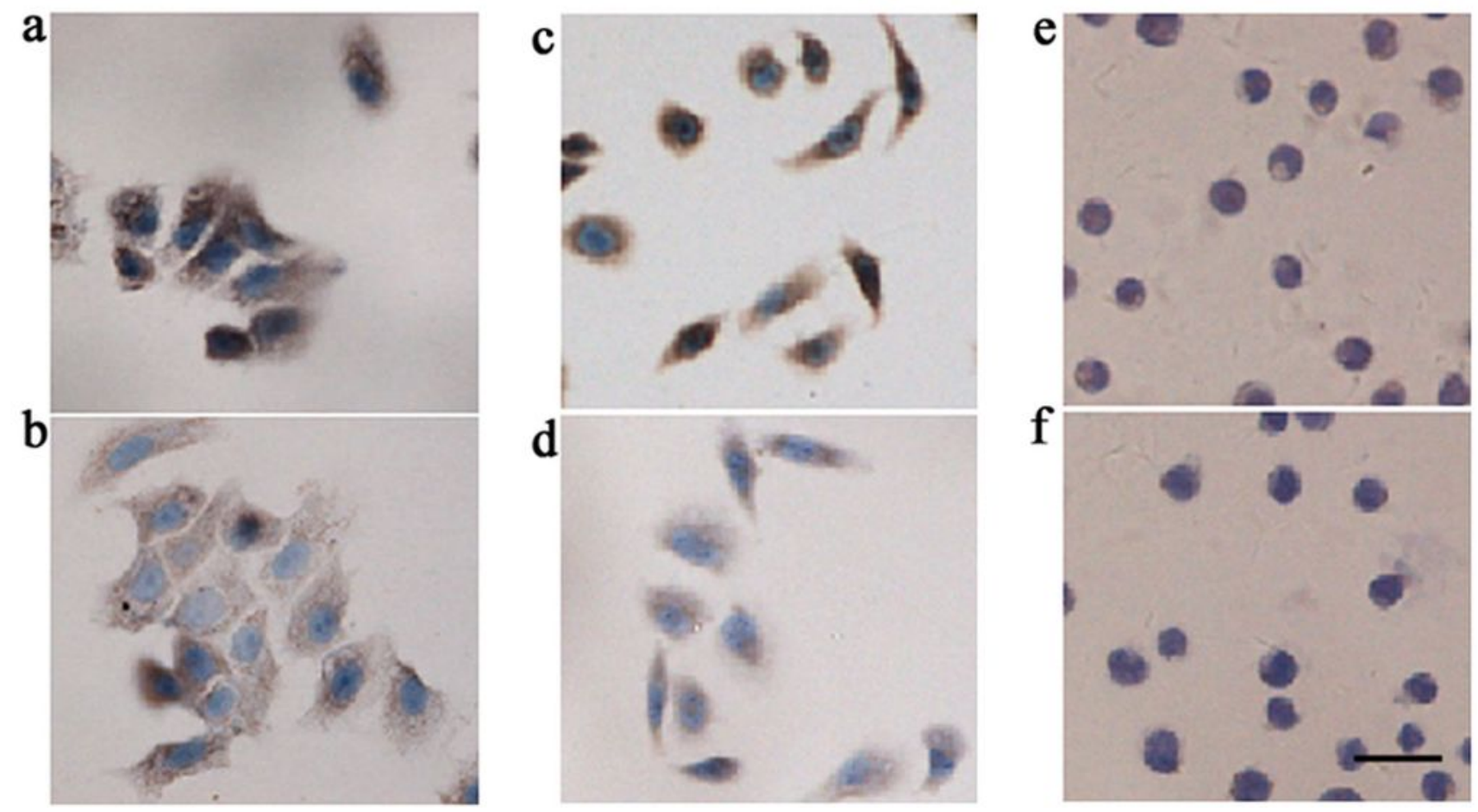

Figure 1

Immunocytochemical detection of CD147 and Lewis y antigen expression in ovarian cancer cells $(\times 400)$. (A) CD147. (B) Lewis y antigen. a: RMG-I-H; b: RMG-l; c: HO8910/PM; d: H08910; e: COC1/DDP; f: COC1. Scale bar: $50 \mu \mathrm{m}$. 


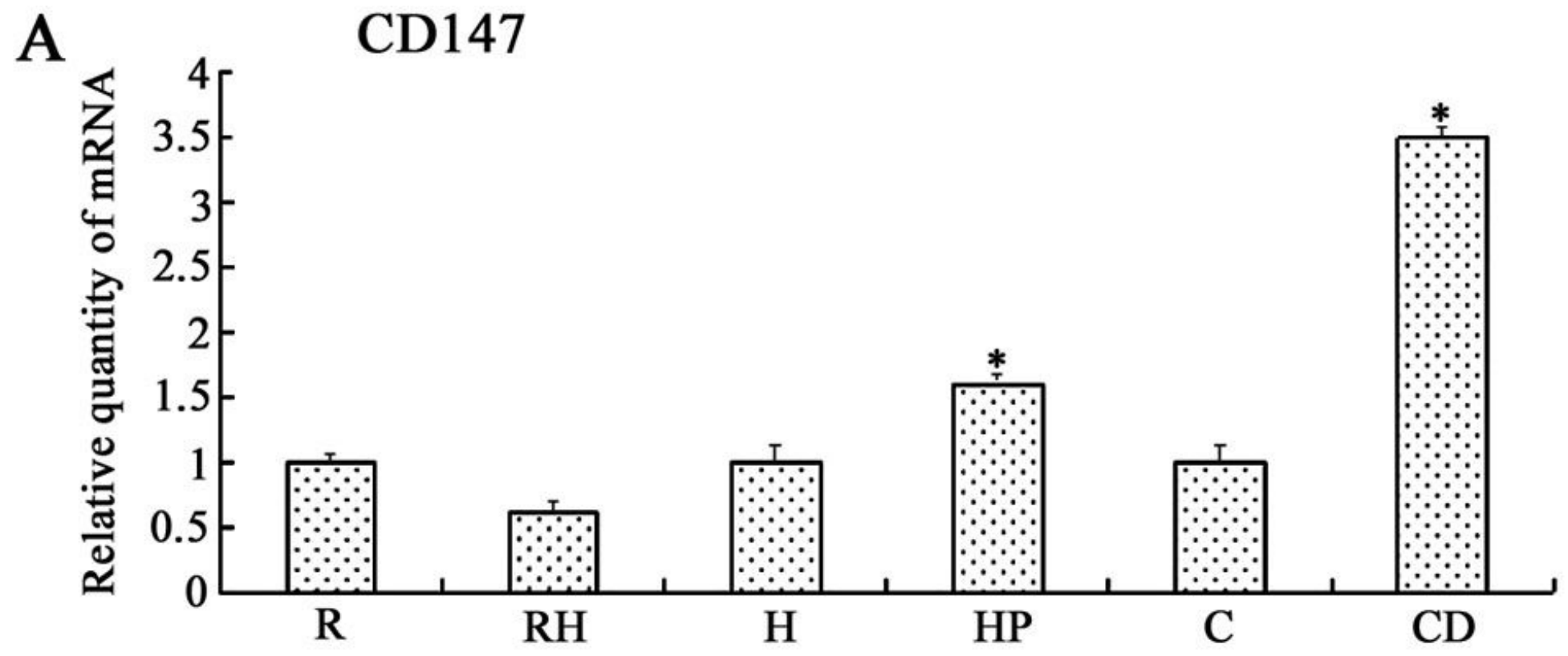

B

FUT1

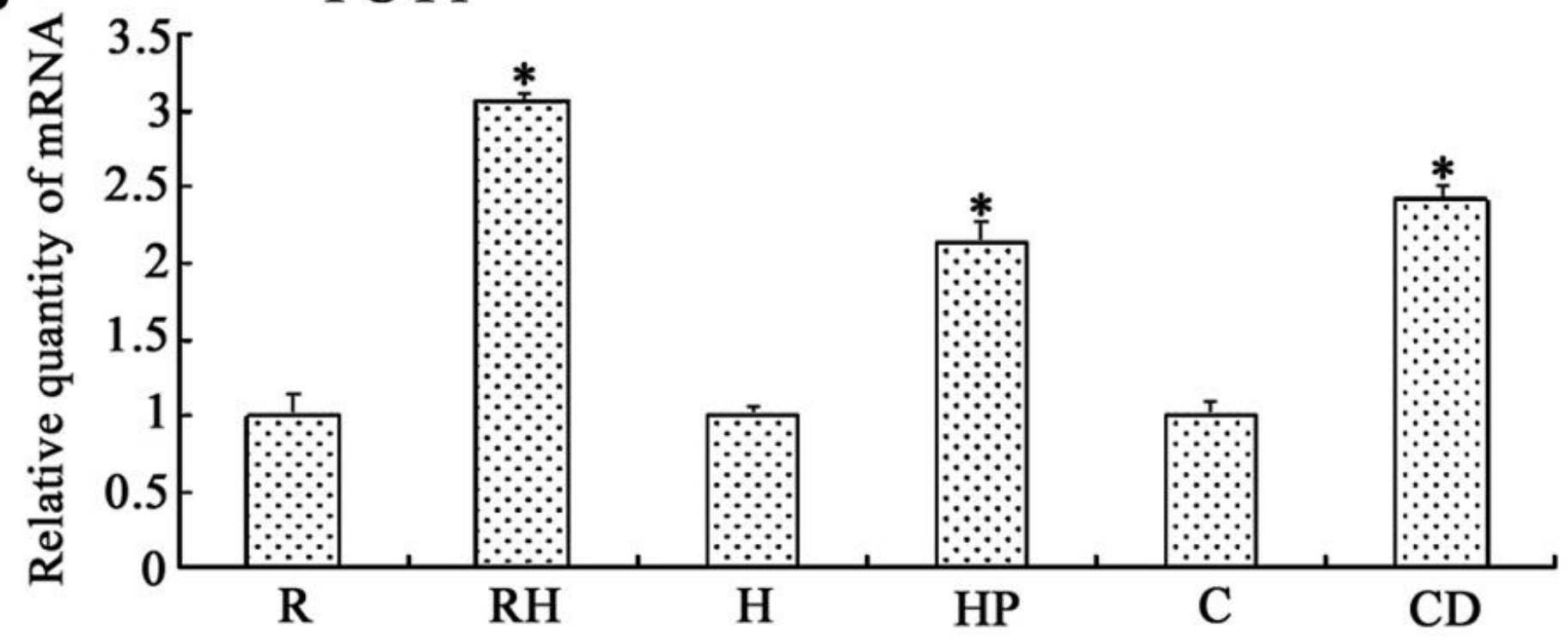

Figure 2

PCR analysis of the expression of CD147 mRNA and FUT1 mRNA in ovarian cancer cells. (A) CD147 mRNA. (B) FUT1 mRNA. *P<0.05, RMG-I-H vs. RMG-I cells, H08910/PM vs. H08910 cells, and COC1/DDP vs. COC1 cells. Bars are labeled as follows: R, RMG-I cells; RH, RMG-I-H cells; H, H08910 cells; HP, H08910/PM cells; C, COC1 cells; CD, COC1/DDP cells. 

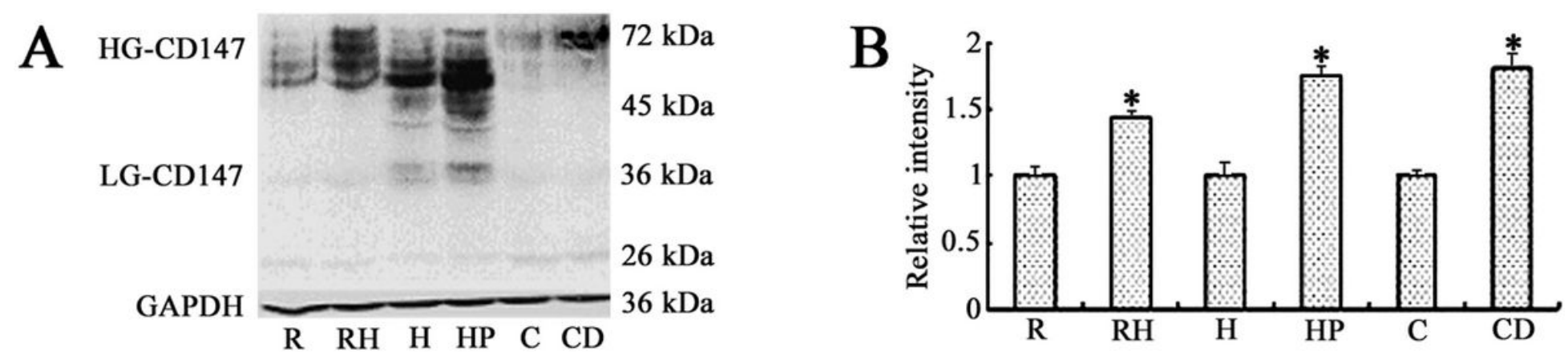

Figure 3

Western blot analysis of the expression of CD147 in ovarian cancer cells. (A) Representative western blots of CD147 in cell lines. (B) Densitometric quantification of protein expression of a $(n=3)$. ${ }^{*}<0.05$, RMG-I-H vs. RMG-I cells, H08910/PM vs. H08910 cells, and COC1/DDP vs. COC1 cells. Bars are labeled as follows: HG-CD147, highly glycosylated form; LG-CD147, low glycosylated form; R, RMG-I cells; RH, RMG-I-H cells; H, H08910 cells; HP, H08910/PM cells; C, COC1 cells; CD, COC1/DDP cells.

A

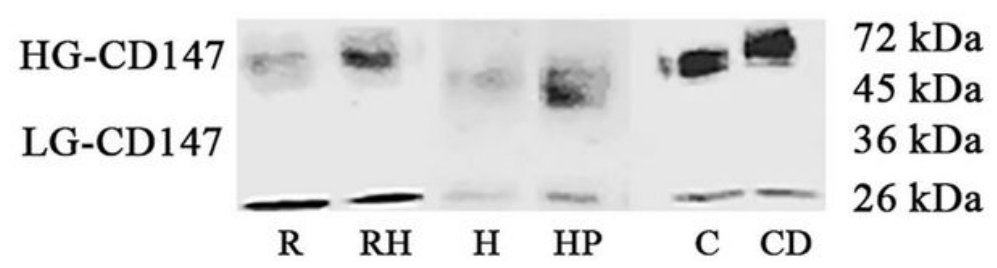

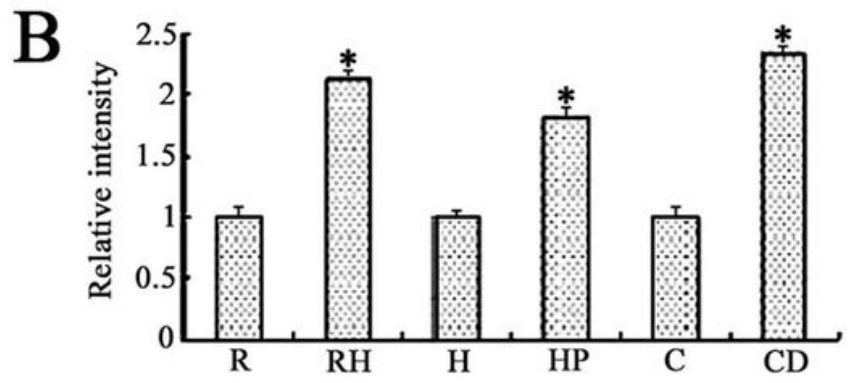

Figure 4

Co-expression of CD147 and Lewis y antigen in ovarian cancer cells. (A) Representative western blots of Lewis y in cell lines. (B) Densitometric quantification of protein expression of a $(n=3)$. ${ }^{*}<<0.05$, RMG-I-H vs. RMG-I cells, H08910/PM vs. H08910 cells, and COC1/DDP vs. COC1 cells. Bars are labeled as follows: HG-CD147, highly glycosylated form; LG-CD147, low glycosylated form; R, RMG-I cells; RH, RMG-IH cells; H, H08910 cells; HP, H08910/PM cells; C, COC1 cells; CD, COC1/DDP cells. 


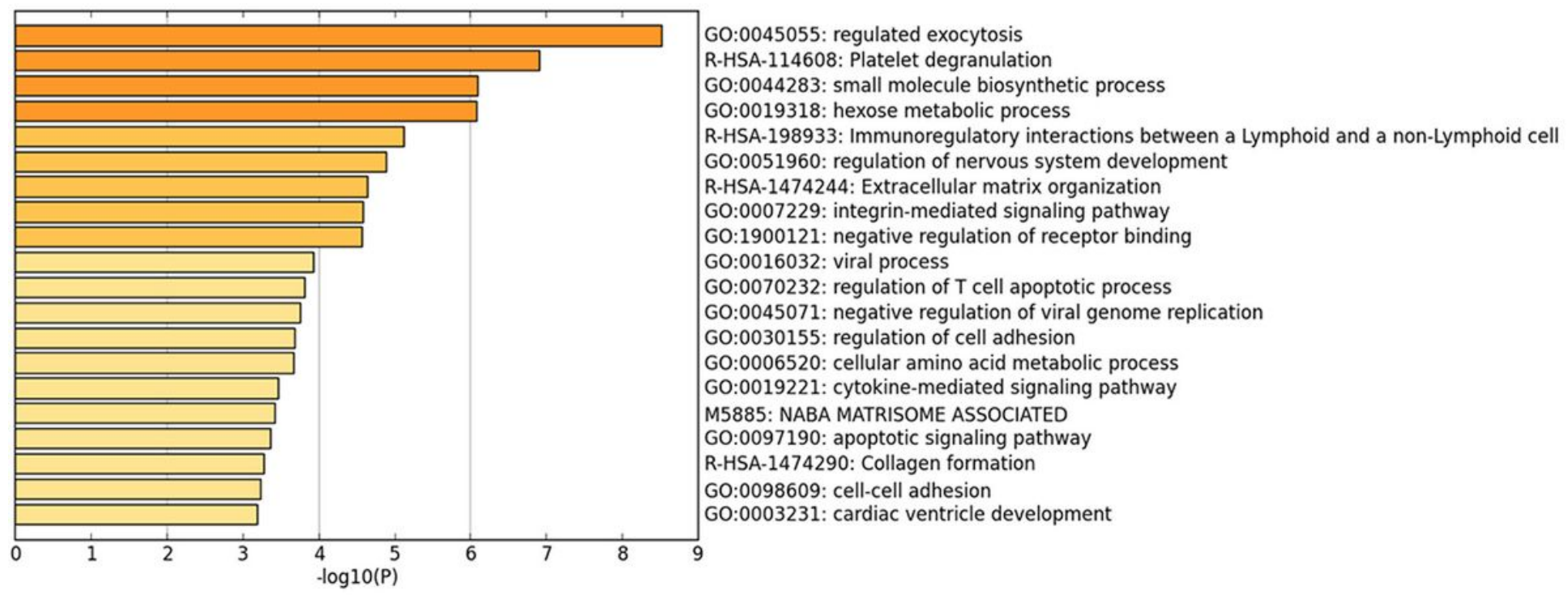

\section{Figure 5}

Analysis of CD147 involved in the differentially expressed genes in response to Lewis y. (Functional enrichment analysis histogram. The vertical coordinate represents the pathway or function name, and the horizontal coordinate corresponds to the number of genes enriched to the region; colors indicate statistical significance. The darker the color, the smaller the $\mathrm{P}$ value. )

A

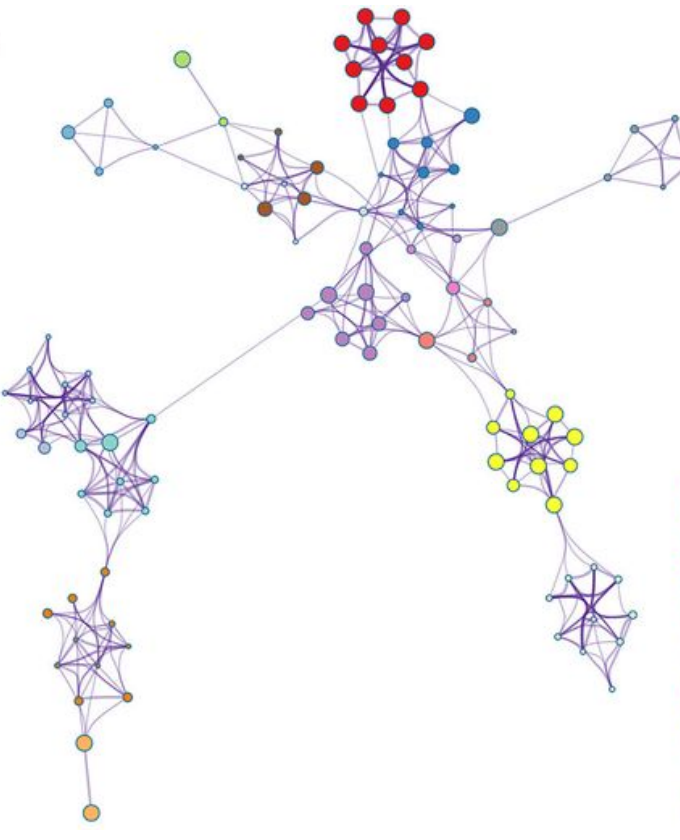

B

Eregulated exocytosis
- Platelet degranulation - ${ }_{\text {m small molecule biosynthetic process }}$ - immunoregulatory interactions between a Lymphoid regulation of nervous system development - Extracellular matrix organization inintegrin-mediated signaling pathway
negative regulation of receptor binding viral process
regulation of $\mathrm{T}$ cell apoptotic process regulation of $T$ cell apoptotic process
negative regulation of viral genome replication regulation of cell adhesion cylokine-mediated signaling pathway NABA MATRISOME ASSOCLATED apoptotic signaling pattway celli-cell adhesion cardiac ventricle development

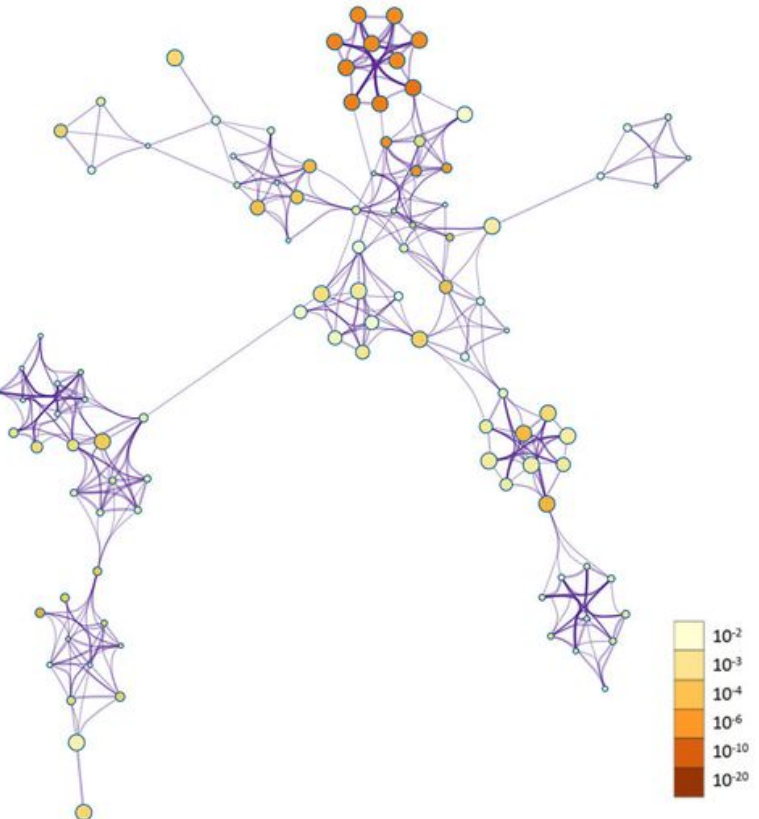

\section{Figure 6}

Genes with a high degree of enrichment were grouped and a network was construced. (A) Colored by cluster ID, nodes sharing the same cluster are typically close to each other; (B) colored by P-value, terms containing more genes tend to have a more significant P-value. 


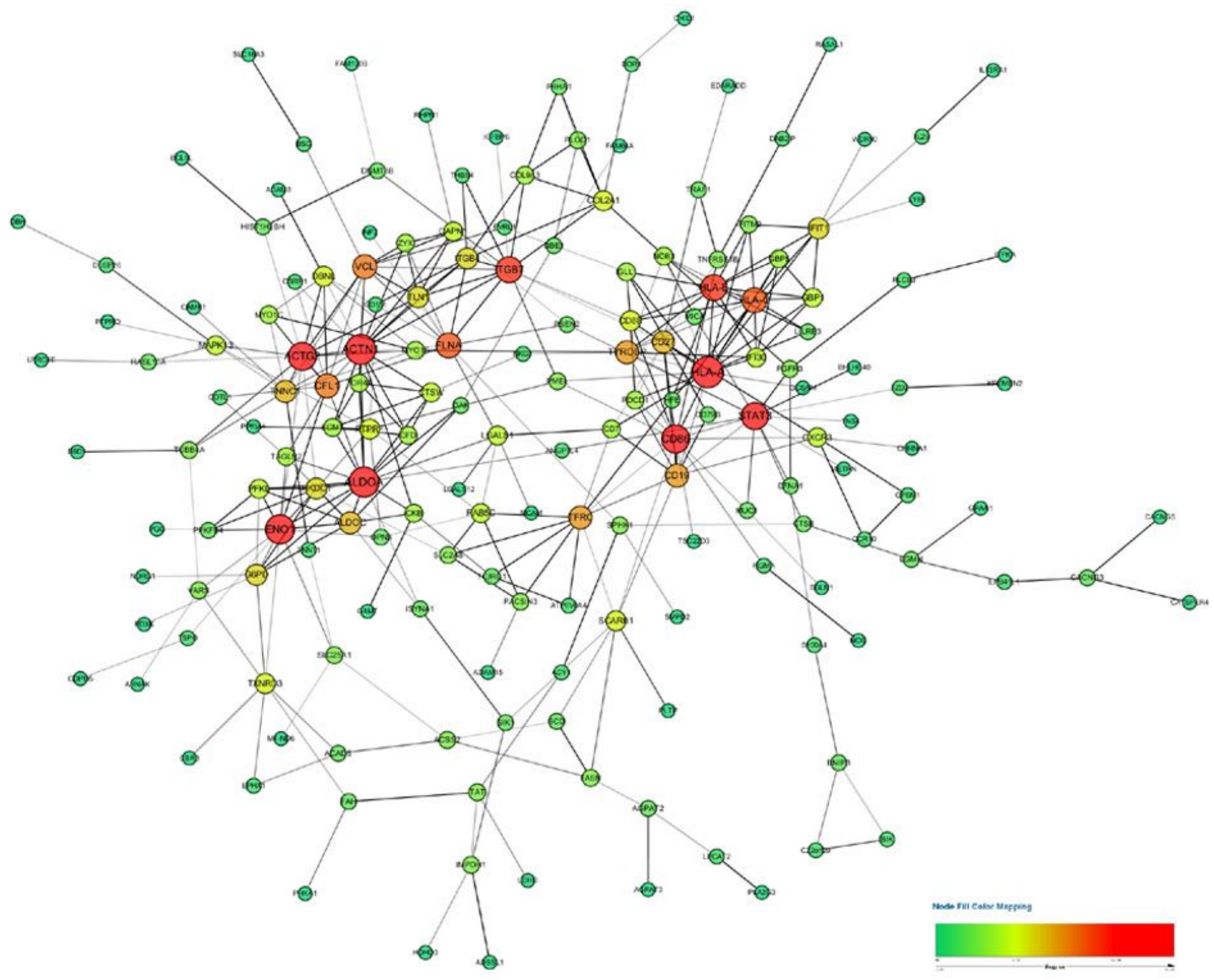

\section{Figure 7}

Protein-protein interaction network of differentially expressed genes. (Each node represents a protein, and the edge between the nodes represents the interaction between the two proteins. The different thickness of the line represents the strength of the relationship. Increased connectivity is represented by a larger the node and more intense red color) 
ALDOA

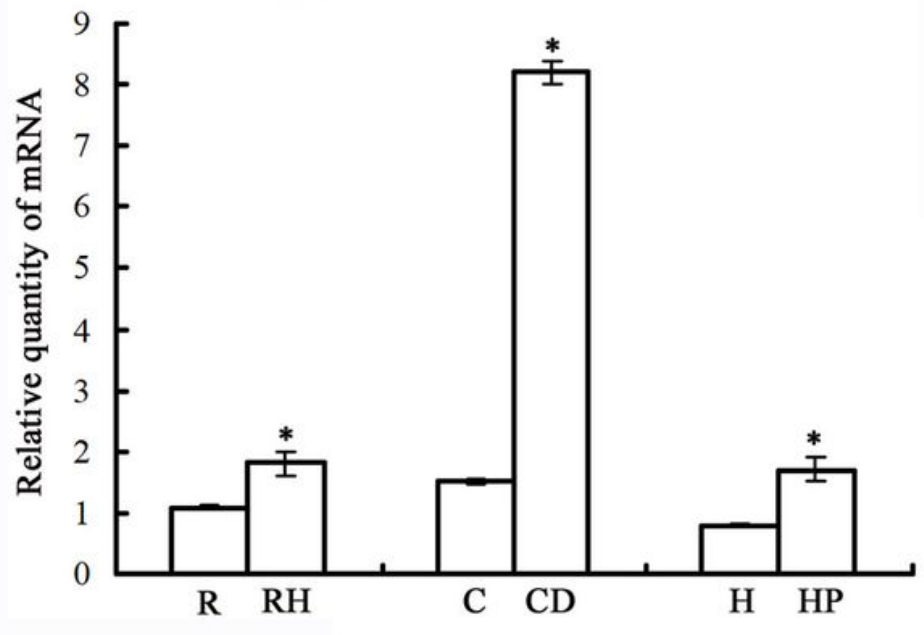

STAT3

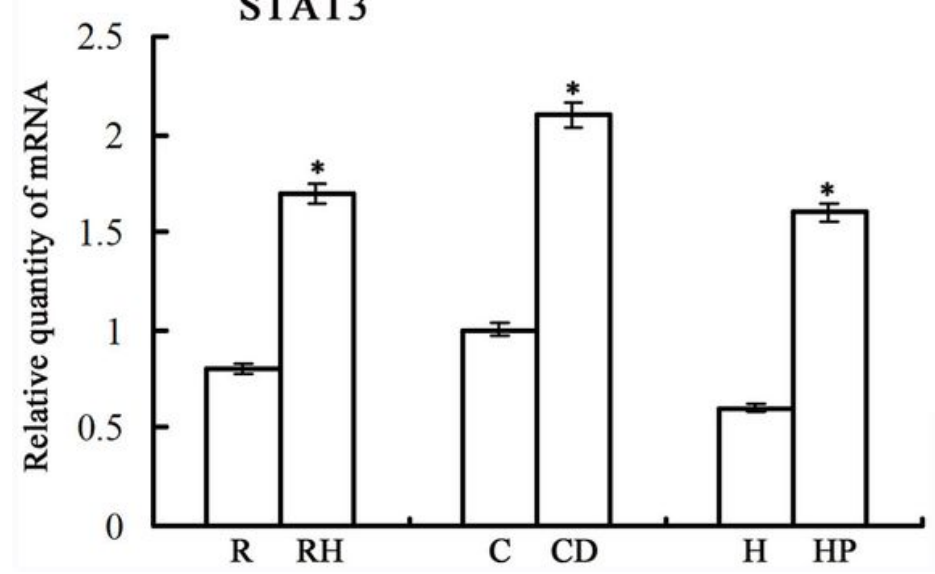

ENO1

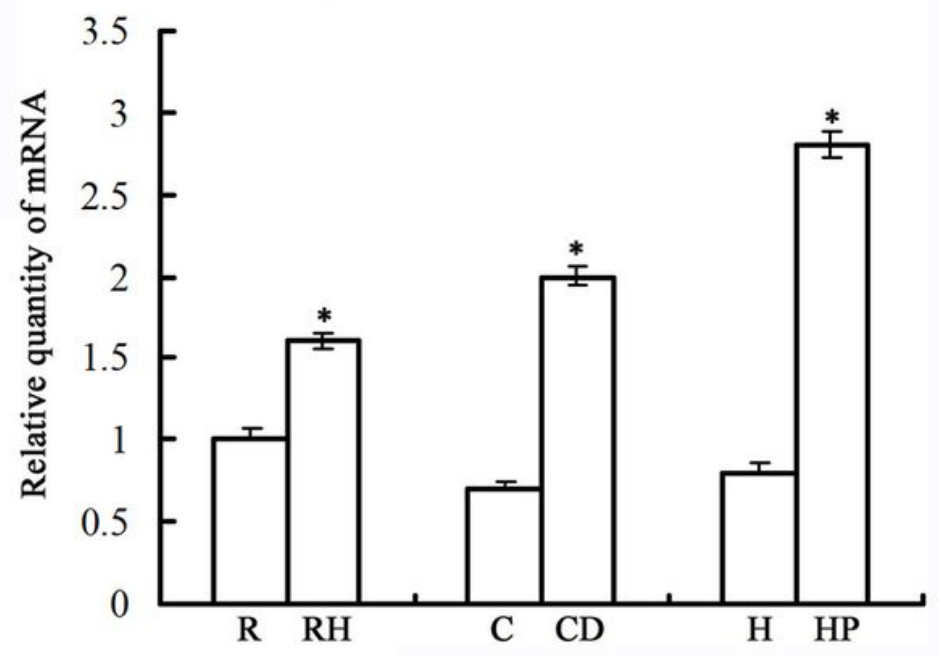

IL1A

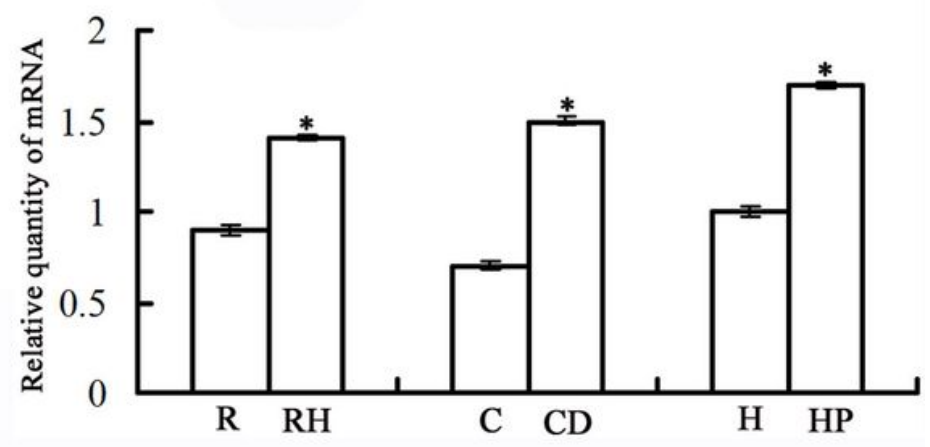

Figure 8

Validation of the differentially expressed genes. (The results of quantitative real-time PCR revealed that the mRNA expression levels of four selected genes (ALDOA, ENO1, STAT3, and IL1A) exhibited obvious differences among the three groups of ovarian cancer cells with varying degrees of malignancy (RMG-I-H, RMG-I, COC1/DDP, COC1, H08910/PM, and H08910 cells). *P<0.05, RMG-I-H vs. RMG-I cells, H08910/PM vs. H08910 cells, and COC1/DDP vs. COC1 cells. Bars are labeled as follows: R, RMG-I cells; RH, RMG-I-H cells; H, H08910 cells; HP, H08910/PM cells; C, COC1 cells; CD, COC1/DDP cells.) 

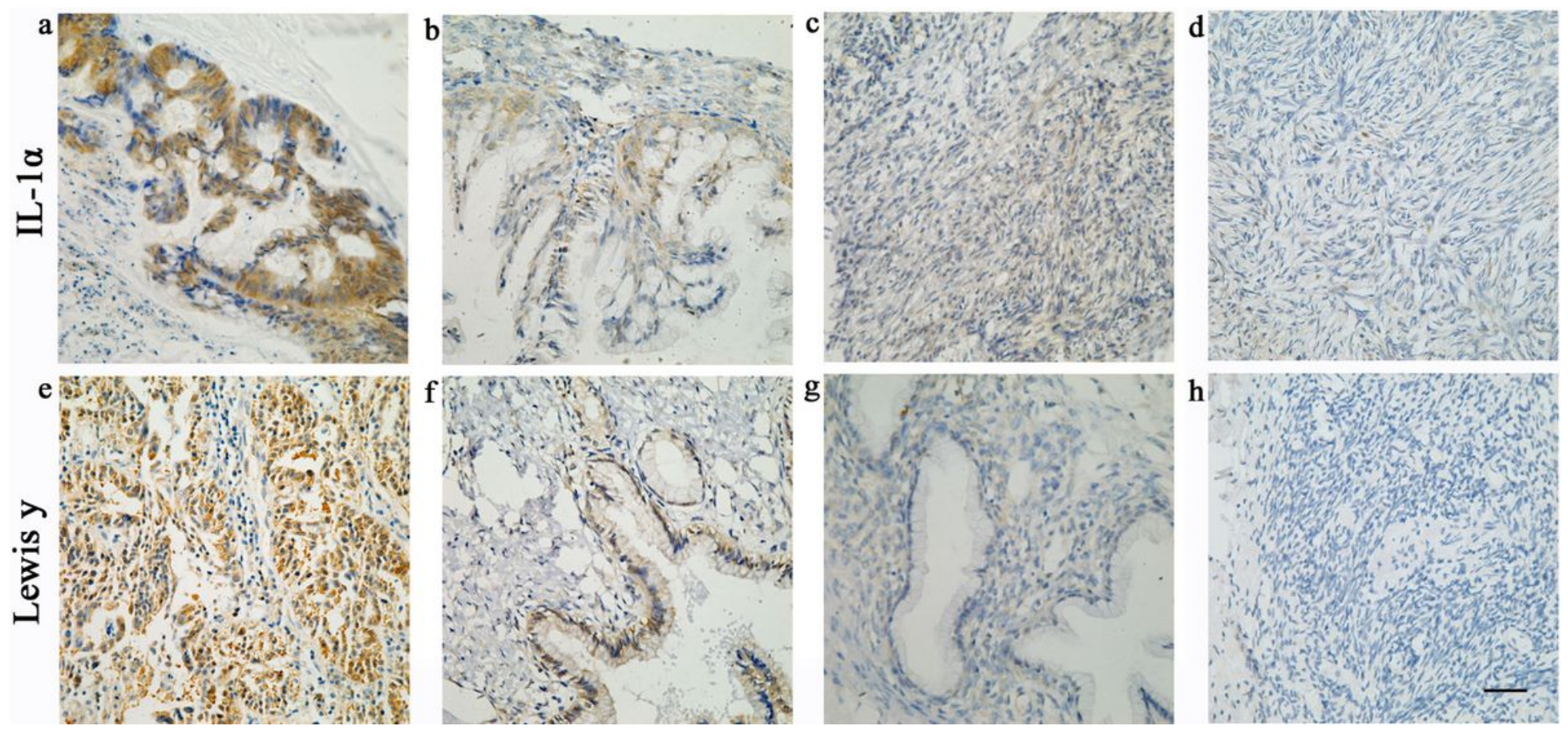

\section{Figure 9}

Immunohistochemical staining of an ovarian malignant tumor $(a, e)$, borderline tumor $(b, f)$, benign tumor $(c, g)$, and normal ovarian tissue (d, h). IL-1a (a, b, c, and d) and Lewis y (e, f, g, and h; original magnification $\times 400)$. Scale bar: $50 \mu \mathrm{m}$. 